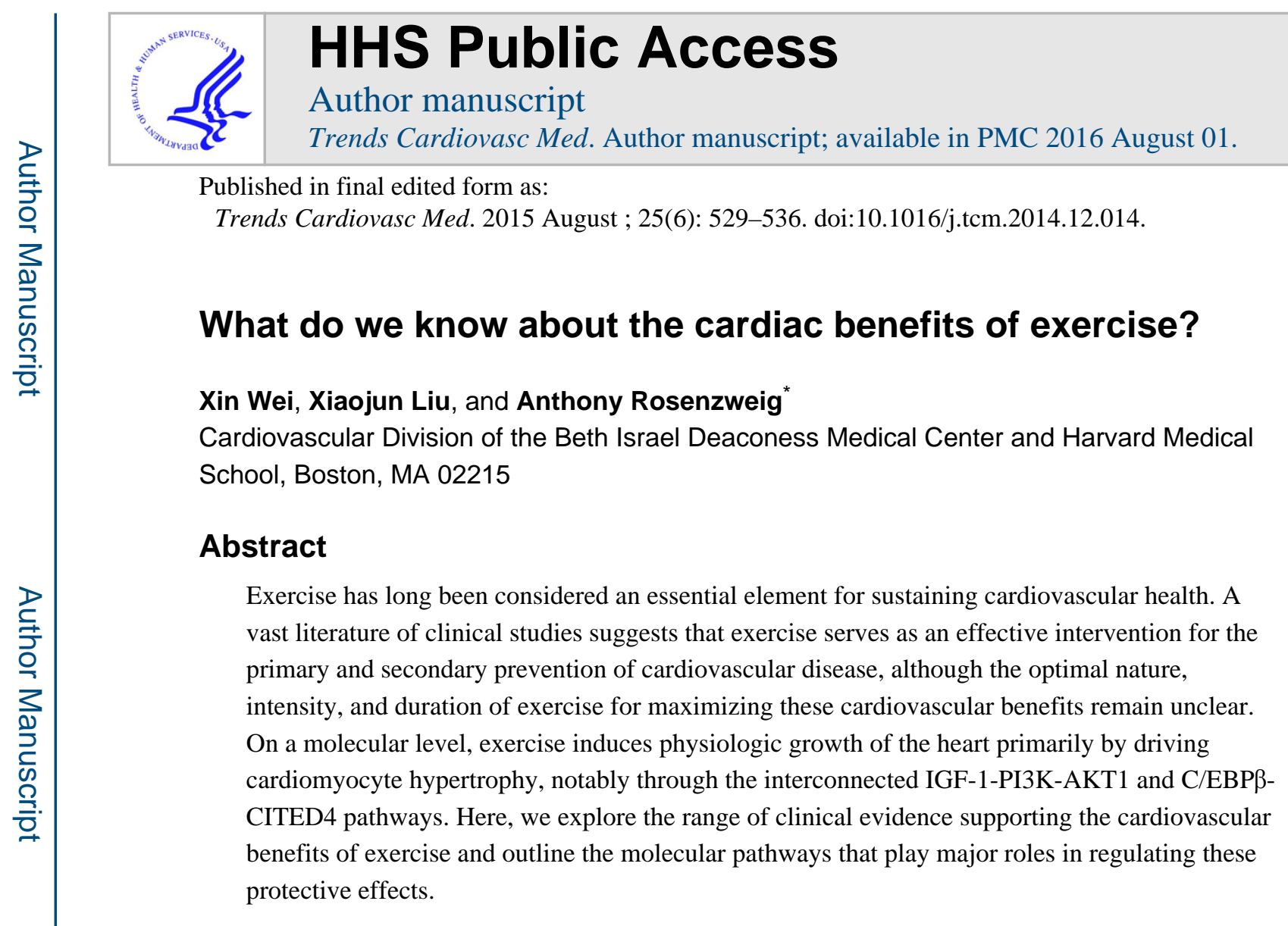

\title{
Introduction
}

For thousands of years, exercise has been thought to play an important role in maintaining one's health. In particular, exercise is believed to be a cheap and effective intervention for the prevention and treatment of heart disease. However, it was not until 1953 that the first scientific study was published describing the potential benefits of exercise on cardiovascular health. In this study, Morris et al. discovered that street car conductors and postmen in London have lower rates of coronary heart disease and overall mortality than street car drivers and desk-based civil servants, respectively [1]. It was hypothesized that these differences were consequences of the greater physical exertion required of conductors who walked the double-decker buses collecting tickets and active postmen who walked around the city delivering mail, compared to that of the sedentary drivers and desk-workers. Although there might have been confounding factors that were not fully controlled for in this study, such as the considerable stress of driving in London traffic or working as a civil servant, many subsequent clinical trials and epidemiological studies have confirmed the benefits of habitual physical activity in preventing cardiovascular disease. Paralleling these clinical findings, recent laboratory studies have delineated several molecular pathways that may play important roles in modulating these clinical phenotypes. In this review, we will

\footnotetext{
" To whom correspondence should be addressed at: Dr. Anthony Rosenzweig, Cardiovascular Division, Beth Israel Deaconess Medical Center, Center for Life Sciences, 3 Blackfan Circle, CLS/947, Boston, MA 02115, arosenzw@ bidmc.harvard.edu.

Publisher's Disclaimer: This is a PDF file of an unedited manuscript that has been accepted for publication. As a service to our customers we are providing this early version of the manuscript. The manuscript will undergo copyediting, typesetting, and review of the resulting proof before it is published in its final citable form. Please note that during the production process errors may be discovered which could affect the content, and all legal disclaimers that apply to the journal pertain.
} 
first describe the effects of exercise on primary and secondary cardiovascular disease prevention, followed by a brief examination of the main molecular pathways involved in regulating the exercise phenotype, and conclude with the therapeutic implications of this research and the directions which we believe this field will be heading in the near future.

\section{Clinical Benefits of Exercise}

Most of the evidence regarding the association between exercise and primary cardiovascular disease prevention comes from observational epidemiological studies (Table). In part this reflects the logistical challenges that would be associated with conducting a sufficiently powered primary prevention trial of exercise as well as the difficulty in ensuring long-term adherence to such an intervention. Nevertheless, the association between habitual exercise and reduced cardiovascular mortality is well documented. An early study found that physical fitness is an excellent predictor of cardiovascular mortality, with lower levels of physical fitness correlating with higher mortality risk from cardiovascular diseases in clinically healthy males [2]. A subsequent study not only confirmed this, but also suggested that even moderate levels of fitness may be able to protect both males and females against the influence of other adverse risk factors for mortality, including smoking and hypertension [3]. Although the distinction is not always made clear, lack of exercise and a sedentary lifestyle appear to be separable risk factors for cardiovascular disease, as shown in various prospective studies [4]. Additionally, low levels of exercise and high sedentary time independently increase the risk of heart failure (HF), even after controlling for socioeconomic, clinical, and other lifestyle risk factors [5]. Although these studies are consistent with the hypothesis that exercise - or physical activity more generally - reduces the risk of a range of cardiovascular diseases, they obviously cannot establish a causal relationship. Since subjects in these observational studies self-select to exercise or not, it remains possible that they do so on the basis of genetic or other differences that are actually the causes for their reduction in adverse cardiovascular outcomes. Moreover, residual unrecognized confounding is always a possibility in observational studies, and exercise is likely a marker for other healthy behaviors. Thus, although an important role for exercise in the primary prevention of cardiovascular disease is plausible and intuitively appealing, it is hard to assert with certainty.

Interventional trials are more feasible for secondary prevention. A large randomized controlled trial (RCT), HF-ACTION, showed that for HF patients, exercise training resulted in significantly improved self-reported health status and moderate reductions in several clinical endpoints, including all-cause mortality or hospitalization, significant when adjusted for highly prognostic predictors of the primary end points [6]. Similarly, the GOSPEL study, a multicenter RCT in Italy, showed that a lifestyle intervention that included exercise after myocardial infarction (MI) led to no significant changes in the primary end points such as cardiovascular mortality, nonfatal MI, or hospitalization for heart failure, but moderately significant reductions in several secondary endpoints, including nonfatal MI, cardiovascular mortality plus nonfatal MI, and cardiovascular mortality plus nonfatal MI and stroke [7]. It is uncertain whether the lack of the clear-cut survival benefit in RCTs reflects an inadequate exercise regimen or statistical power, imperfect adherence, or the inability of exercise to mitigate mortality in these settings. 
To overcome the statistical limitations of small trials, there have been numerous metaanalyses of RCTs that assessed the effects of exercise (Table). One study found that aerobic exercise training reduced adverse left ventricular remodeling in HF patients, supplemental to established pharmacological treatment, while strength training showed no demonstrable benefit [8]. A meta-analysis of 34 RCTs found that exercise-based cardiac rehabilitation after MI was associated with reductions in mortality and rates of reinfarction, with even relatively short-term exercise-based cardiac rehabilitation protocols potentially translating into long-term benefits [9]. A common worry among patients with established cardiovascular disease is whether the stress of exercise will cause further damage to their heart. Results from the ExTraMATCH collaborative meta-analysis found that properlysupervised exercise training programs for HF patients are not dangerous, but may actually reduce overall mortality [10].

The secondary preventative effects of exercise are also prominent in other relevant disease populations. A meta-analysis of RCTs involving diabetes patients found that exercise reduces the risk of cardiovascular disease in these patients [11]. Similarly, a prospective study found that in adults with hypertension, higher levels of cardiorespiratory fitness might be able to offset the mortality risk associated with higher adiposity [12]. Most interestingly, a recent meta-epidemiological study suggested that the mortality benefit of exercise in coronary heart disease or prediabetes patients was not statistically different from that seen with established drug interventions [13]. Even more strikingly, the authors claimed that among stroke patients, exercise interventions were more effective than drug treatment such as anticoagulants and antiplatelet agents at reducing mortality [13], although one suspects that other endpoints (e.g. recurrent stroke) might more readily have revealed differences. Regardless of whether these statements can be proven in randomized controlled trials, a wealth of current clinical evidence drawn from observational studies, RCTs, and metaanalyses supports the concept that exercise is indeed an effective intervention for the prevention of many diseases, cardiovascular and otherwise.

Given this, the American Heart Association (AHA), in conjunction with the American College of Cardiology (ACC), recommends that healthy adults engage in moderate-intensity cardiorespiratory exercise training for at least 30 minutes per day, 5 days per week, for a total of 150 minutes per week. However, a strong evidence base supporting these - or any other - specific recommendations is generally lacking. In addition to the challenges of demonstrating unequivocally the health benefits of exercise discussed above, there are even less data available to define the optimal nature, intensity, and duration of exercise regimens to achieve these benefits [14]. Interestingly, a recent observational study suggested that even running at moderate speeds for an average of five minutes a day was associated with reductions in cardiovascular and all-causes mortality, with no clear additional benefit associated with greater running duration or intensity [15].

As with most physiologic stimuli, exercise may become deleterious if practiced at excessive levels. The incidence of cardiac arrest is three to five times more likely during marathons compared to half-marathons [16]. Several studies of elite athletes have also found that prolonged, excessive endurance exercise can be associated with maladaptive cardiac phenotypes including fibrosis [17], adverse left ventricular remodeling [18] and atrial 
fibrillation [19, 20], although confounding or even reverse causality (subjects with concerns about their cardiovascular health may take up extreme exercise in some cases) cannot be fully excluded. For atrial fibrillation, animal studies provide additional support for the hypothesis that prolonged exercise can lead to increased fibrosis, diastolic dysfunction, and atrial fibrillation [21, 22], at least partially driven by angiotensin II activation [23]. For postMI patients, while increasing exercise generally reduces further cardiovascular diseaserelated mortality, excessive exercise (running $>7.1 \mathrm{~km} /$ day or briskly walking $>10.7 \mathrm{~km} /$ day) may be associated with increased cardiovascular disease-related mortality [24]. It seems likely that exercise should be practiced in moderation to maximize its cardiovascular and systemic benefits, although the precise boundaries are poorly defined and may vary among individuals.

\section{Molecular Mechanisms}

The plasticity of the heart allows for adaptations to the increases in cardiopulmonary demand imposed by exercise. These adaptations include left ventricular hypertrophy and improved $\mathrm{Ca}^{2+}$ handling, which improves cardiac function. Systemically, vascular changes, such as angiogenesis, and metabolic changes, including improved insulin sensitivity and metabolism, help the heart and body maintain metabolic homeostasis and reduce the risks of cardiovascular diseases. The following reviews provide more thorough examinations of the phenotypic adaptations that promote the clinical benefits of exercise [25, 26]. Here, we will focus primarily on the molecular mechanisms that underlie these phenotypic changes.

In response to the increased cardiac demand of endurance exercise, the heart undergoes physiologic remodeling and hypertrophy. While physiological cardiac hypertrophy due to exercise and pathological cardiac hypertrophy due to hypertension or aortic stenosis are difficult to distinguish clinically, recent studies have shown that exercise and other physiological stimuli induce a transcriptional network distinct from that seen with pathological stimuli [27]. Additionally, although some have suggested that the distinctions between physiological and pathological cardiac hypertrophy are simply due to the intermittent nature of the pressure overload on the heart by physiological stimuli, compared to the more persistent stress by pathological stimuli, an interesting study found that applying intermittent pathological pressure to the heart results in a milder, but nevertheless pathological hypertrophic response [28]. Furthermore, while exercise-induced physiological cardiac hypertrophy is typically accompanied by proportional increases in the length and width of cardiomyocytes, pathological cardiac hypertrophy often shows disproportionate increases in cardiomyocyte length. Taken together these studies support the hypothesis that physiological and pathological cardiac hypertrophy are mechanistically distinct processes even at a stage when the hearts are structurally and functionally indistinguishable.

Exercise-induced physiological cardiac growth may reflect a combination of cellular hypertrophy and hyperplasia. An intensive swimming protocol in mice can induce up to a $45 \%$ increase in the cardiomyocyte size in as little as two weeks, and it seems likely that changes in cardiomyocyte size are the dominant contributor to overall physiological heart growth, particularly over short periods of time [27]. However, exercise induces proliferation of at least some cell types in the heart, such as endothelial cells. Intriguingly, recent work by 
our group and others has suggested that exercise increases markers of proliferation in cardiomyocytes, once thought to be stagnant and terminally differentiated after maturation. Independent work now suggests that cardiomyocyte renewal does occur naturally at low levels in the adult heart in both animals and humans $[29,30]$ but little is known about the physiological regulators of this process. We found that exercise increases markers of proliferation in the cardiomyocyte lineage, and identified a transcriptional pathway that appears to mediate this effect (Figure). This pathway works through the CCAAT/enhancerbinding protein $\beta$ (C/EBP $\beta)$ [27] and CITED4 (CBP/p300-Interacting Transactivators with $\mathbf{E}$ (glutamic acid)/D (aspartic acid)-rich-carboxylterminal domain [31]). Cardiac C/EBP $\beta$ decreases with exercise, and genetically mimicking this change in vivo recapitulates many of the phenotypes seen in exercised hearts and protects mice from transverse aortic constriction (TAC)-induced heart failure [27]. CITED4 increases in exercised hearts (or with genetic reduction in $\mathrm{C} / \mathrm{EBP} \beta$ ). In neonatal rat cardiomyocytes (NRVMs) in vitro, CITED4 is necessary and sufficient for proliferation [27], although neonatal cardiomyocytes are known to retain more capacity for proliferation than adult cardiomyocytes. Of note, the increase in proliferation markers seen in vivo is specifically in cells expressing cardiomyocyte sarcomere proteins, which could be cardiomyocytes or committed cardiac progenitor cells (CPC), which appear to express sarcomere proteins at some stages of development [32]. Consistent with the latter model, a subsequent study suggests that exercise training increases the number of putative cardiac progenitor cells in the $\mathrm{c}-\mathrm{kit}^{\mathrm{pos}}$ or Sca- ${ }^{\text {pos }}$ lineages, giving rise to new cardiomyocytes [33]. However, it is important to note that none of these studies can prove that exercise drives cardiomyocyte proliferation in vivo. Unambiguously identifying the source and fate of any new cardiomyocytes formed, as well as whether these cells survive and functionally integrate into the myocardium, is of great interest but will require long-term fate-mapping experiments to address.

Interestingly, exercise has been reported to drive cellular proliferation and a potentially regenerative response in multiple other tissues. Like the heart, the brain was once thought to be terminally differentiated and incapable of generating new neurons after early development. However, multiple studies have now shown that exercise can stimulate neurogenesis and cellular proliferation in the dentate gyrus region of the brain [34]. Similarly, exercise was found to induce an increase in the number of hematopoietic stem cells in the bone marrow [35] and to cause proliferation of myogenic stem cells in skeletal muscle [36]. Taken together, these data suggest that exercise induces a proliferative and potentially regenerative response by providing fundamental inductive signals that are conserved across multiple cell lineages. We believe that identifying and learning to exploit these signals holds great promise for regenerative medicine in multiple contexts.

Some of the molecular signals mediating the benefits of exercise have already been identified. Exercise training has a demonstrable cardioprotective effect, reducing cardiomyocyte apoptosis after an ischemic insult [37]. Exercise also offsets the age-induced increase in $\mathrm{Bax} / \mathrm{Bcl} 2 \mathrm{a}$ ratios, leading to a reduction in cardiomyocyte apoptosis and cardiac remodeling in aging rats [38]. In addition to alterations in pro- and anti-apoptotic Bcl-family member ratios, many effects of exercise are mediated by the IGF-1 or neuregulin-1 (NRG-1) activation of PI3K-AKT1 signaling pathway (Figure). Physiological cardiac hypertrophy 
can be induced by IGF-1 administration, which also protects the heart against myocardial ischemia-reperfusion injury [39]. On a molecular level, IGF-1 binds to its tyrosine-kinase receptor, IGF1-R, which activates the p110a subunit of phosphoinositide-3 kinase (PI3K). PI3K (p110a) is necessary and sufficient for the induction of physiological hypertrophy [40], and also protects the heart against MI- or TAC-induced HF [41]. Exercise-induced activation of IGF-1 and PI3K then leads to the activation of the serine-threonine kinase, AKT1 (V-akt murine thymoma viral oncogene homolog 1), which is also necessary [42] and sufficient for physiological cardiac growth. In contrast, inhibition of PI3K (p110a) does not affect pathological hypertrophy due to pressure overload [40] and genetic deletion of AKT1 in fact accelerates pathological hypertrophy in this setting, suggesting AKT1 actually inhibits pathological cardiac growth after TAC [42]. Of note, AKT1 expression in cardiomyocytes also downregulates C/EBP $\beta$ expression which, as noted above, is sufficient to recapitulate a number of exercise-induced cardiac phenotypes [27].

Endurance exercise also plays an important role in the induction of blood vessels in the heart needed to support the increased metabolic demands and cardiac growth. Exercise induces the release of circulating catecholamines, such as epinephrine and norepinephrine, which targets beta-adrenergic receptors, activating endothelial nitric oxide synthase (eNOS). The activated eNOS releases nitric oxide (NO) into the blood stream, which helps to modulate vascular tone, smooth muscle proliferation, angiogenesis, and reduces myocardial fibrosis. NO homeostasis plays a crucial role in regulating the cardioprotective effects of exercise, particularly against damages caused by ischemia-reperfusion injury [43]. Interestingly, AKT1 also promotes activation of eNOS through phosphorylation [44].

\section{MicroRNAs}

Recent studies suggest that microRNAs (miRNAs, miRs) are major regulators of gene expression in the heart. MiRNAs are short $\sim 22$ nucleotide strands of non-coding RNA that bind to the $3^{\prime}$ or $5^{\prime}$ untranslated region (UTR) of their target genes and downregulate the protein expression of these target genes. MiRNAs play significant roles in cardiac pathophysiology [45], cardiovascular development [46], and cardiac regeneration [47]. Several studies have begun to characterize the regulation and role of miRNAs in the exercised heart. A recent investigation profiled the global expression pattern of cardiac miRNAs in physiological left ventricular hypertrophy [48]. A mechanistic study found that aerobic exercise could increase levels of miR-29 in the heart, suppressing collagen gene expression and leading to improved ventricular compliance and other beneficial cardiac effects [49]. Interestingly, exercise alters miRNAs that regulate the PI3K-AKT1 pathway, helping to drive the exercise-induced physiological cardiac response [50].

Circulating miRNAs may also be particularly relevant to the physiological exercise response. These miRNAs are secreted or released from cells into the plasma of an animal and may serve important roles in intercellular communication, potentially regulating both the cells that release them and the cells that take them up. We believe circulating miRNAs may be especially intriguing in the exercise response because they could potentially help drive similar phenotypes, such as cellular proliferation, across multiple organ systems. Of particular interest are miRNAs circulating in exosomes, which pass through the blood-brain- 
barrier under certain conditions, and could in theory transmit exercise-induced signals contributing to the beneficial effects of exercise on the brain. Circulating miRNAs may also serve as clinically useful biomarkers for cardiovascular fitness and/or risk, due to their stability and specificity [51]. As an example, miR 423-5p was recently reported as a circulating biomarker for heart failure [52].

Recent efforts have tried to profile changes in circulating miRNAs in response to aerobic exercise in humans and found distinct circulating miRNA signatures following acute and sustained exercise [53, 54]. Additional human studies assessed the distinct changes in circulating miRNA levels under various exercise modalities [55], and also examined the unique temporal dynamic regulation of specific circulating miRNAs after prolonged exercise [56]. These studies may help identify potential biomarkers for assessing levels of cardiovascular fitness in patients, paving the way for more targeted counseling and personalized treatments for heart disease patients in the clinic. More studies will be needed to validate not only the precise profile of circulating miRNAs after exercise but also to investigate their functional roles.

MiRNAs also have the potential to be exploited for therapeutic purposes. Recently, modified nucleotides such as locked-nucleic acid (LNA) miRNA inhibitors (antimiRs) and agonists (agomiRs) have been developed for use in vivo models and early-phase clinical trials. LNAantimiRs have been used to treat HCV infection by downregulating miR-122 in clinical trials and appeared to be generally well-tolerated [57]. Another study found that miR-214 agomiR targeting ATF4 could inhibit bone formation [58]. These studies and many others highlight the therapeutic potential of miRNAs to treat disease. By identifying miRNAs that contribute to the benefits of exercise, we may one day be able to manipulate these miRNAs in the clinic to restore cardiovascluar health to patients with heart disease.

\section{Future directions}

Since much is still unknown about the mechanisms underlying the exercise-induced physiologic changes in the heart, there are numerous research directions that will be interesting to pursue. Now that we are beginning to clinically distinguish the risks associated with a sedentary life-style and lack of habitual exercise, it would be interesting to assess whether there may be distinct molecular pathways that modulate these processes. Furthermore, a more precise understanding of the time and intensity-response of exercise, as well as the mechanisms that drive these responses, may help us uncover novel, functionallyrelevant pathways that play important roles in promoting cardiovascular health and preventing adverse cardiac remodeling. This may be an area of synergy between clinical and animal studies, where it is easier to mandate and maintain exercise protocols. However, extrapolating results from animals to humans is itself non-trivial given differences in species physiology and scale. Undoubtedly, it will also be valuable to more fully characterize the pathways that regulate cardiomyogenesis in the adult heart, and in this context, exercise may provide a useful model of a physiological inductive cue that we hypothesize enhances the endogenous reparative capacity of the heart. While much is known about the roles of miRNAs in regulating pathological or developmental cardiac pathways, we know less about their functional roles in modulating the exercise-induced cardiac response. It would be 
interesting to identify miRNAs altered in the heart and plasma by exercise, and elucidate the molecular pathways that are regulated by these miRNAs. In this context, it may also be valuable to compare the miRNA profiles of exercised human and animals, in order to help develop appropriate and applicable animal models to elucidate the molecular mechanisms underlying their function in exercise. Ultimately, understanding the mechanisms that these miRNAs play in the physiologic exercise response may lead to the development of miRNA therapies that can help prevent primary and secondary onset of cardiovascular diseases and restore heart health to patients. Lastly, another fascinating area of exploration may be to assess the role of exercise in modulating the physiological responses of aging. While much has been suggested regarding the potential anti-aging effects of exercise, very little is known about the molecular mechanisms linking exercise and aging. A clearer understanding of this relationship on a basic science level may lead to the identification of novel therapeutic targets, which could one day help to stall or reverse the adverse effects of aging and agingrelated diseases.

\section{Why should we care?}

Physical inactivity is a major risk factor for disease burden worldwide. A wealth of clinical data suggest that exercise is one of the cheapest and most effective interventions for promoting cardiovascular health, so those who can safely exercise should. However, not everyone is physically capable of exercising and ironically those most in need (e.g. those with established cardiovascular disease) are often those for whom exercise training is most challenging. It is worth remembering (and reminding our patients) that even modest levels of physical activity are associated with considerable health benefits. Nevertheless, elucidation of the molecular mechanisms mediating the protective effects of exercise may lead to the identification of novel biomarkers and/or therapeutic targets, not previously gleaned from complementary studies of disease. It is also possible that small molecules or biological interventions aimed at these targets could optimize specific aspects of the cardiovascular exercise response in a way difficult or impossible to achieve through exercise, although we are skeptical that any drug intervention or "exercise mimetic" will fully recapitulate the myriad benefits of habitual physical activity.

\section{Acknowledgments}

This research was supported by grants from the NIH (AR[R21HL114352, 1UH2TR000901, R01HL110733, R01HL122987], XL[T32HL073734]. XW was supported by the Harvard College Research Program and the Herchel Smith-Harvard Research Fellowship. AR is a principal faculty member of the Harvard Stem Cell Institute.

\section{References}

1. Morris JN, et al. Coronary heart-disease and physical activity of work. Lancet. 1953; 265(6795): 1053-7. contd. [PubMed: 13110049]

2. Ekelund LG, et al. Physical fitness as a predictor of cardiovascular mortality in asymptomatic North American men. The Lipid Research Clinics Mortality Followup Study. N Engl J Med. 1988; 319(21):1379-84. [PubMed: 3185648]

3. Blair SN, et al. Influences of cardiorespiratory fitness and other precursors on cardiovascular disease and all-cause mortality in men and women. JAMA. 1996; 276(3):205-10. [PubMed: 8667564]

4. Proper KI, et al. Sedentary behaviors and health outcomes among adults: a systematic review of prospective studies. Am J Prev Med. 2011; 40(2):174-82. [PubMed: 21238866] 
5. Young DR, et al. Effects of physical activity and sedentary time on the risk of heart failure. Circ Heart Fail. 2014; 7(1):21-7. [PubMed: 24449810]

6. O'Connor CM, et al. Efficacy and safety of exercise training in patients with chronic heart failure: HF-ACTION randomized controlled trial. JAMA. 2009; 301(14):1439-50. [PubMed: 19351941]

7. Giannuzzi $P$, et al. Global secondary prevention strategies to limit event recurrence after myocardial infarction: results of the GOSPEL study, a multicenter, randomized controlled trial from the Italian Cardiac Rehabilitation Network. Arch Intern Med. 2008; 168(20):2194-204. [PubMed: 19001195]

8. Haykowsky MJ, et al. A meta-analysis of the effect of exercise training on left ventricular remodeling in heart failure patients: the benefit depends on the type of training performed. J Am Coll Cardiol. 2007; 49(24):2329-36. [PubMed: 17572248]

9. Lawler PR, Filion KB, Eisenberg MJ. Efficacy of exercise-based cardiac rehabilitation postmyocardial infarction: a systematic review and meta-analysis of randomized controlled trials. Am Heart J. 2011; 162(4):571-584 e2. [PubMed: 21982647]

10. Piepoli MF, et al. Exercise training meta-analysis of trials in patients with chronic heart failure (ExTraMATCH). BMJ. 2004; 328(7433):189. [PubMed: 14729656]

11. Kodama S, et al. Association between physical activity and risk of all-cause mortality and cardiovascular disease in patients with diabetes: a meta-analysis. Diabetes Care. 2013; 36(2):4719. [PubMed: 23349151]

12. McAuley PA, et al. The joint effects of cardiorespiratory fitness and adiposity on mortality risk in men with hypertension. Am J Hypertens. 2009; 22(10):1062-9. [PubMed: 19617881]

13. Naci H, Ioannidis JP. Comparative effectiveness of exercise and drug interventions on mortality outcomes: metaepidemiological study. BMJ. 2013; 347:f5577. [PubMed: 24473061]

14. Lee IM, Skerrett PJ. Physical activity and all-cause mortality: what is the dose-response relation? Med Sci Sports Exerc. 2001; 33(6 Suppl):S459-71. discussion S493-4. [PubMed: 11427772]

15. Lee DC, et al. Leisure-time running reduces all-cause and cardiovascular mortality risk. J Am Coll Cardiol. 2014; 64(5):472-81. [PubMed: 25082581]

16. Kim JH, et al. Cardiac arrest during long-distance running races. N Engl J Med. 2012; 366(2):13040. [PubMed: 22236223]

17. Wilson M, et al. Diverse patterns of myocardial fibrosis in lifelong, veteran endurance athletes. $\mathbf{J}$ Appl Physiol (1985). 2011; 110(6):1622-6. [PubMed: 21330616]

18. La Gerche A, et al. Exercise-induced right ventricular dysfunction and structural remodelling in endurance athletes. Eur Heart J. 2012; 33(8):998-1006. [PubMed: 22160404]

19. O'Keefe JH, et al. Potential adverse cardiovascular effects from excessive endurance exercise. Mayo Clin Proc. 2012; 87(6):587-95. [PubMed: 22677079]

20. Karjalainen J, et al. Lone atrial fibrillation in vigorously exercising middle aged men: case-control study. BMJ. 1998; 316(7147):1784-5. [PubMed: 9624065]

21. Benito B, et al. Cardiac arrhythmogenic remodeling in a rat model of long-term intensive exercise training. Circulation. 2011; 123(1):13-22. [PubMed: 21173356]

22. Guasch E, et al. Atrial fibrillation promotion by endurance exercise: demonstration and mechanistic exploration in an animal model. J Am Coll Cardiol. 2013; 62(1):68-77. [PubMed: 23583240]

23. Gay-Jordi G, et al. Losartan prevents heart fibrosis induced by long-term intensive exercise in an animal model. PLoS One. 2013; 8(2):e55427. [PubMed: 23383330]

24. Williams PT, Thompson PD. Increased cardiovascular disease mortality associated with excessive exercise in heart attack survivors. Mayo Clin Proc. 2014; 89(9):1187-94. [PubMed: 25128072]

25. Mann N, Rosenzweig A. Can exercise teach us how to treat heart disease? Circulation. 2012; 126(22):2625-35. [PubMed: 23184282]

26. Baggish AL, Wood MJ. Athlete's heart and cardiovascular care of the athlete: scientific and clinical update. Circulation. 2011; 123(23):2723-35. [PubMed: 21670241]

27. Bostrom $\mathrm{P}$, et al. C/EBPbeta controls exercise-induced cardiac growth and protects against pathological cardiac remodeling. Cell. 2010; 143(7):1072-83. [PubMed: 21183071]

28. Perrino $\mathrm{C}$, et al. Intermittent pressure overload triggers hypertrophy-independent cardiac dysfunction and vascular rarefaction. J Clin Invest. 2006; 116(6):1547-60. [PubMed: 16741575] 
29. Bergmann O, et al. Evidence for cardiomyocyte renewal in humans. Science. 2009; 324(5923):98102. [PubMed: 19342590]

30. Senyo SE, et al. Mammalian heart renewal by pre-existing cardiomyocytes. Nature. 2013; 493(7432):433-6. [PubMed: 23222518]

31. Yahata T, et al. The MSG1 non-DNA-binding transactivator binds to the $\mathrm{p} 300 / \mathrm{CBP}$ coactivators, enhancing their functional link to the Smad transcription factors. J Biol Chem. 2000; 275(12): 8825-34. [PubMed: 10722728]

32. Bailey B, et al. Cardiac stem cell genetic engineering using the alphaMHC promoter. Regen Med. 2009; 4(6):823-33. [PubMed: 19903002]

33. Waring CD, et al. The adult heart responds to increased workload with physiologic hypertrophy, cardiac stem cell activation, and new myocyte formation. Eur Heart J. 2012

34. van Praag H, Kempermann G, Gage FH. Running increases cell proliferation and neurogenesis in the adult mouse dentate gyrus. Nat Neurosci. 1999; 2(3):266-70. [PubMed: 10195220]

35. De Lisio M, Parise G. Characterization of the effects of exercise training on hematopoietic stem cell quantity and function. J Appl Physiol (1985). 2012; 113(10):1576-84. [PubMed: 23019311]

36. Nielsen JL, et al. Proliferation of myogenic stem cells in human skeletal muscle in response to lowload resistance training with blood flow restriction. J Physiol. 2012; 590(Pt 17):4351-61. [PubMed: 22802591]

37. Quindry J, et al. Exercise training provides cardioprotection against ischemia-reperfusion induced apoptosis in young and old animals. Exp Gerontol. 2005; 40(5):416-25. [PubMed: 15919594]

38. Kwak HB, Song W, Lawler JM. Exercise training attenuates age-induced elevation in Bax/Bcl-2 ratio, apoptosis, and remodeling in the rat heart. FASEB J. 2006; 20(6):791-3. [PubMed: 16459353]

39. Buerke M, et al. Cardioprotective effect of insulin-like growth factor I in myocardial ischemia followed by reperfusion. Proc Natl Acad Sci U S A. 1995; 92(17):8031-5. [PubMed: 7644533]

40. McMullen JR, et al. Phosphoinositide 3-kinase(p110alpha) plays a critical role for the induction of physiological, but not pathological, cardiac hypertrophy. Proc Natl Acad Sci U S A. 2003; 100(21):12355-60. [PubMed: 14507992]

41. Lin RC, et al. PI3K(p110 alpha) protects against myocardial infarction-induced heart failure: identification of PI3K-regulated miRNA and mRNA. Arterioscler Thromb Vasc Biol. 2010; 30(4): 724-32. [PubMed: 20237330]

42. DeBosch B, et al. Akt1 is required for physiological cardiac growth. Circulation. 2006; 113(17): 2097-104. [PubMed: 16636172]

43. Calvert JW, et al. Exercise protects against myocardial ischemia-reperfusion injury via stimulation of beta(3)-adrenergic receptors and increased nitric oxide signaling: role of nitrite and nitrosothiols. Circ Res. 2011; 108(12):1448-58. [PubMed: 21527738]

44. Dimmeler $S$, et al. Activation of nitric oxide synthase in endothelial cells by Akt-dependent phosphorylation. Nature. 1999; 399(6736):601-5. [PubMed: 10376603]

45. van Rooij E, et al. A signature pattern of stress-responsive microRNAs that can evoke cardiac hypertrophy and heart failure. Proc Natl Acad Sci U S A. 2006; 103(48):18255-60. [PubMed: 17108080]

46. Zhao Y, et al. Dysregulation of cardiogenesis, cardiac conduction, and cell cycle in mice lacking miRNA-1-2. Cell. 2007; 129(2):303-17. [PubMed: 17397913]

47. Eulalio A, et al. Functional screening identifies miRNAs inducing cardiac regeneration. Nature. 2012; 492(7429):376-81. [PubMed: 23222520]

48. Martinelli NC, et al. An analysis of the global expression of microRNAs in an experimental model of physiological left ventricular hypertrophy. PLoS One. 2014; 9(4):e93271. [PubMed: 24751578]

49. Soci UP, et al. MicroRNAs 29 are involved in the improvement of ventricular compliance promoted by aerobic exercise training in rats. Physiol Genomics. 2011; 43(11):665-73. [PubMed: 21447748]

50. Ma Z, et al. Swimming exercise training-induced left ventricular hypertrophy involves microRNAs and synergistic regulation of the PI3K/AKT/mTOR signaling pathway. Eur J Appl Physiol. 2013; 113(10):2473-86. [PubMed: 23812090] 
51. Creemers EE, Tijsen AJ, Pinto YM. Circulating microRNAs: novel biomarkers and extracellular communicators in cardiovascular disease? Circ Res. 2012; 110(3):483-95. [PubMed: 22302755]

52. Tijsen AJ, et al. MiR423-5p as a circulating biomarker for heart failure. Circ Res. 2010; 106(6): 1035-9. [PubMed: 20185794]

53. Baggish AL, et al. Dynamic regulation of circulating microRNA during acute exhaustive exercise and sustained aerobic exercise training. J Physiol. 2011; 589(Pt 16):3983-94. [PubMed: 21690193]

54. Nielsen $\mathrm{S}$, et al. The miRNA plasma signature in response to acute aerobic exercise and endurance training. PLoS One. 2014; 9(2):e87308. [PubMed: 24586268]

55. Banzet $\mathrm{S}$, et al. Changes in circulating microRNAs levels with exercise modality. J Appl Physiol (1985). 2013; 115(9):1237-44. [PubMed: 23950168]

56. Baggish AL, et al. Rapid upregulation and clearance of distinct circulating microRNAs after prolonged aerobic exercise. J Appl Physiol (1985). 2014; 116(5):522-31. [PubMed: 24436293]

57. Janssen HL, et al. Treatment of HCV infection by targeting microRNA. N Engl J Med. 2013; 368(18):1685-94. [PubMed: 23534542]

58. Wang X, et al. miR-214 targets ATF4 to inhibit bone formation. Nat Med. 2013; 19(1):93-100. [PubMed: 23223004] 


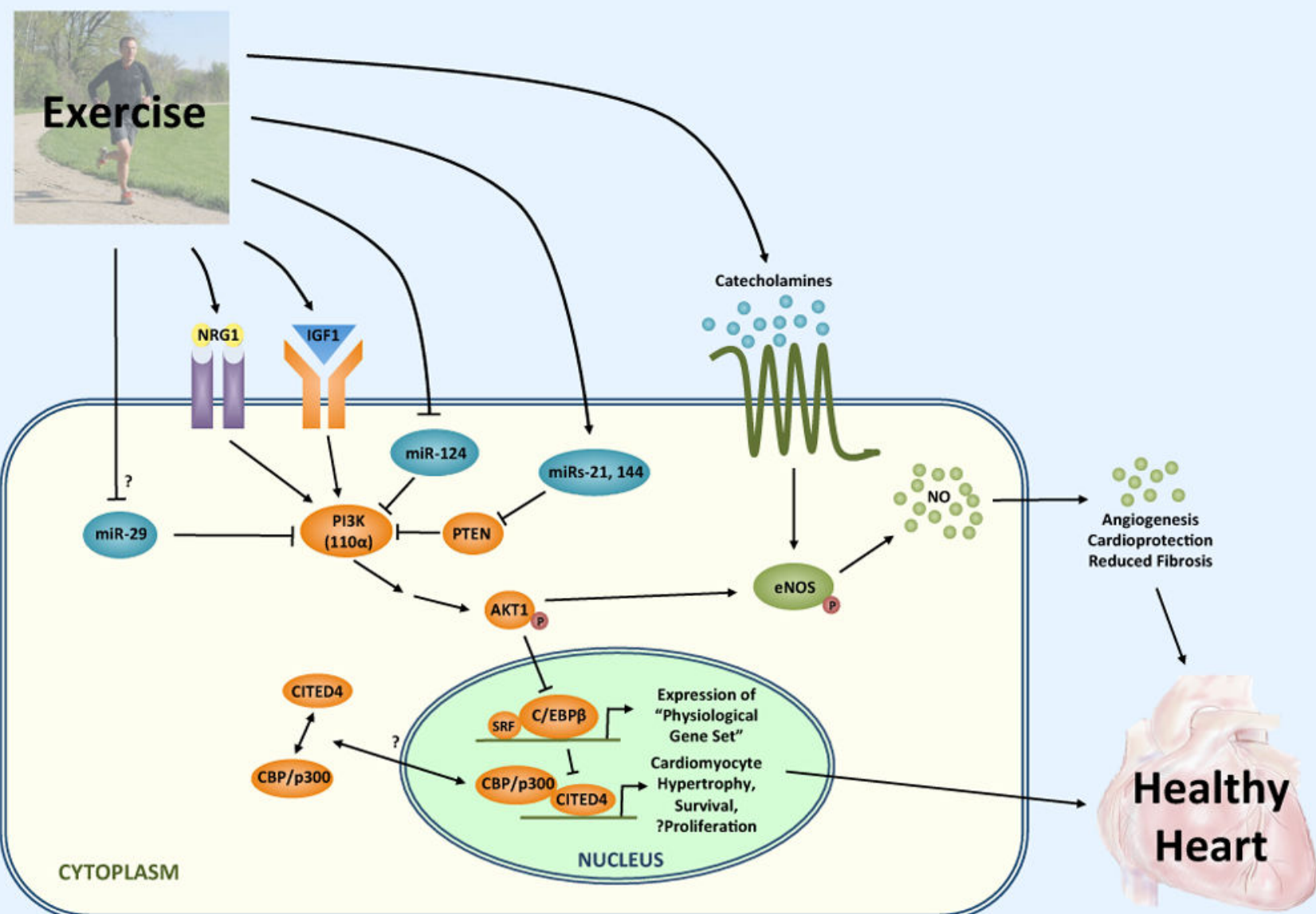

Figure. Representative molecular pathways contributing to the cardiac exercise phenotype Exercise increases both IGF-1 and neuregulin (NRG-1), activating the PI3K-AKT1 pathway, which in turn inhibits the transcription factor $\mathrm{C} / \mathrm{EBP} \beta$ in the nucleus.

Downregulation of $\mathrm{C} / \mathrm{EPB} \beta$ activates the expression of a physiological gene set that drives cardiac growth by cardiomyocyte hypertrophy and proliferation, including upregulation of CITED4. CITED4 binds CBP/p300, either in the cytoplasm or nucleus, and also activates genes involved in cardiomyocyte growth and proliferation. Moreover, exercise leads to the differential expression of specific miRNAs, including some that are upregulated (e.g. miR-21 and miR-144) and others that are downregulated (e.g. miR-124 and potentially miR-29), that target and help regulate various components of IGF-1-PI3K-AKT1 signaling among other pathways. Lastly, exercise-induced release of catecholamines and activation of AKT1 concurrently mediate angiogenesis and other protective vascular remodeling via activation of eNOS. 


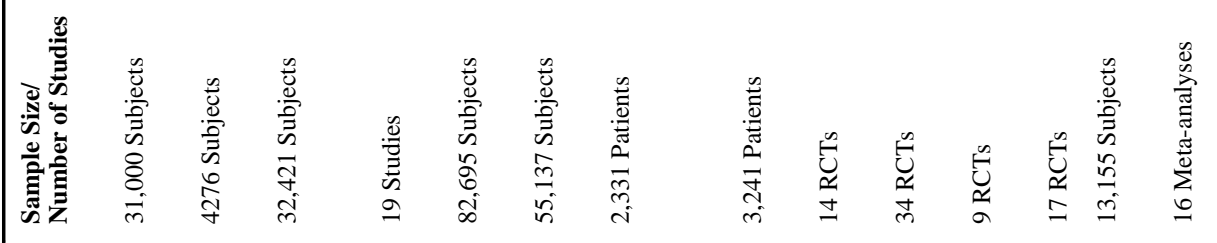

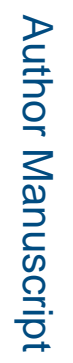
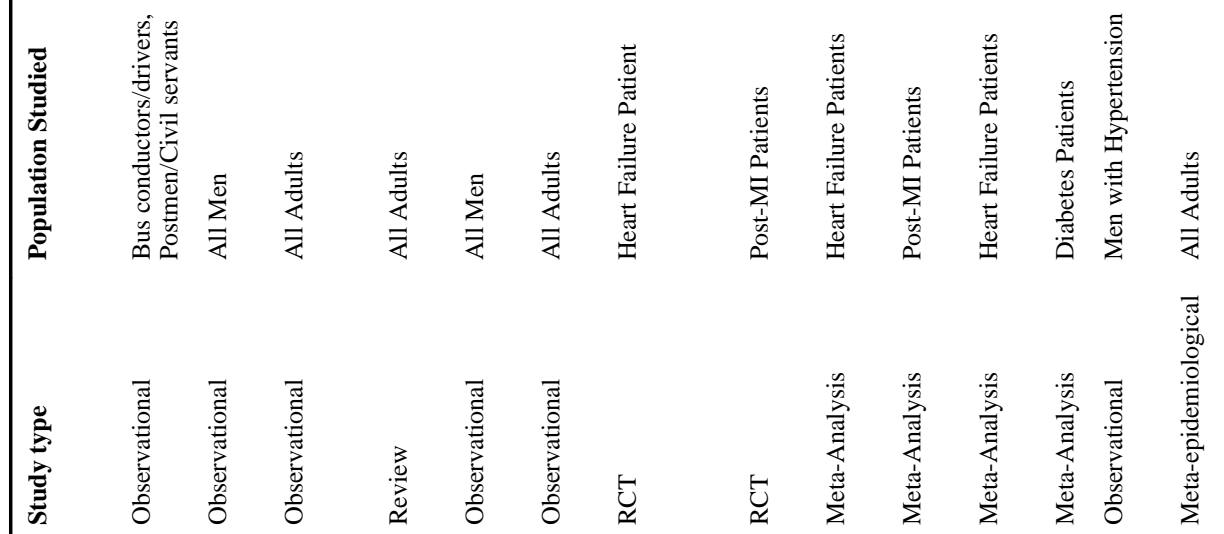

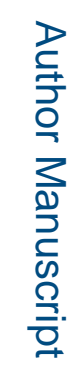

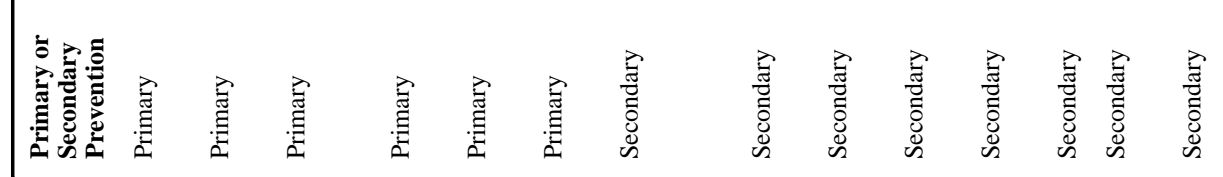

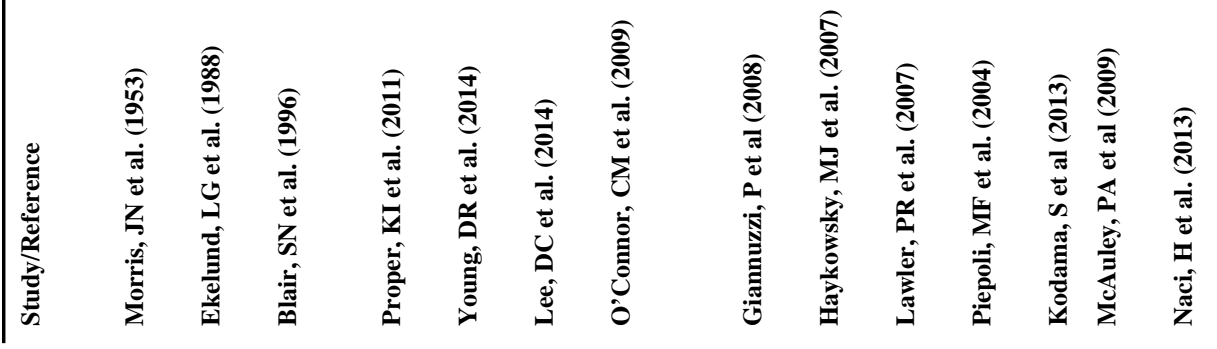

Trends Cardiovasc Med. Author manuscript; available in PMC 2016 August 01. 